Document downloaded from:

http://hdl.handle.net/10251/80121

This paper must be cited as:

Tamaño-Machiavello, MN.; Bracke, B.; Costa, C.; Lanceros-Mendez, S.; Sabater I Serra, R.; Gómez Ribelles, JL. (2016). Hydrophobic/Hydrophilic P(VDF-TrFE)/PHEA Polymer Blend Membranes. Journal of Polymer Science Part B: Polymer Physics. 54:672-679. doi:10.1002/polb.23959.

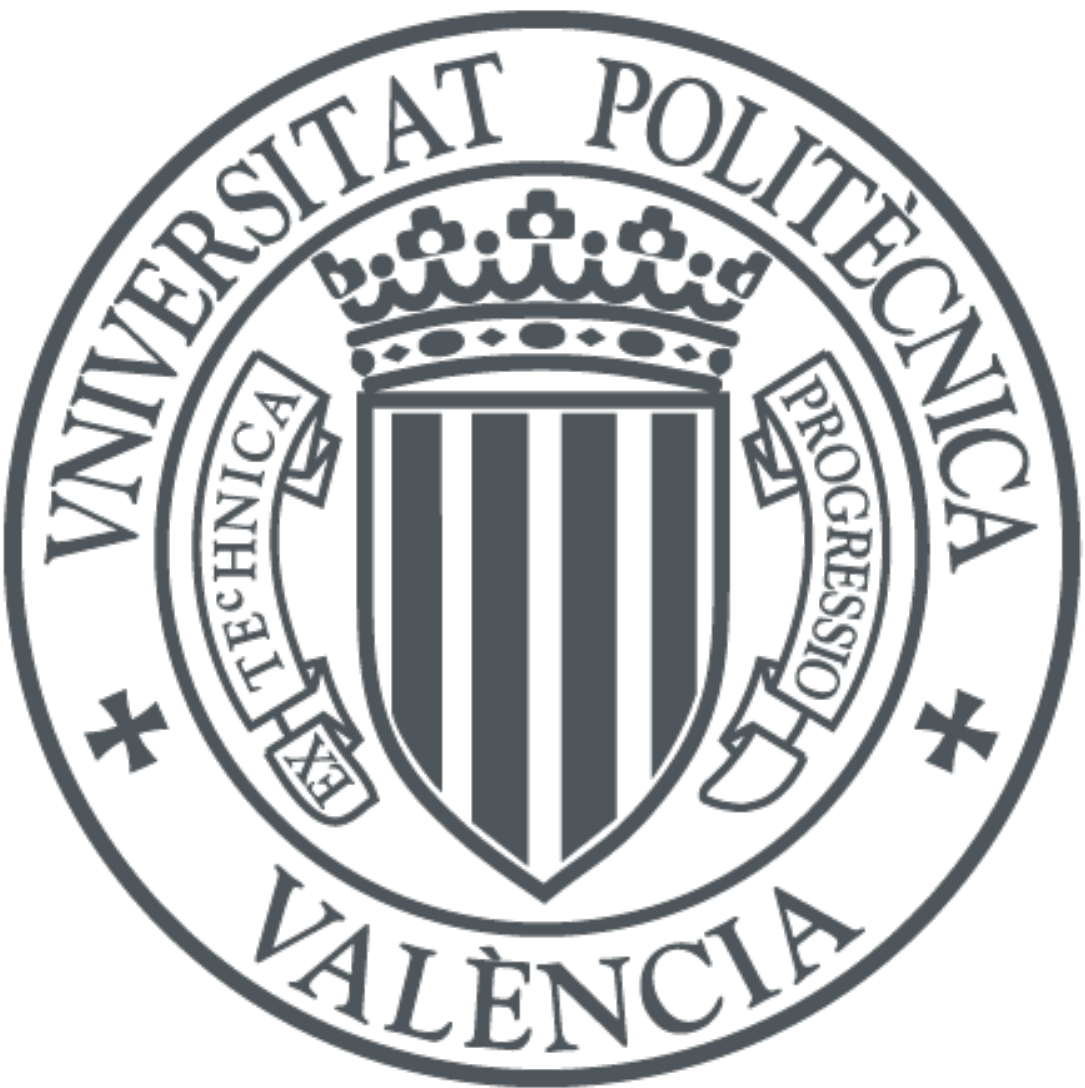

The final publication is available at

http://dx.doi.org/10.1002/polb.23959

Copyright Wiley

Additional Information 


\title{
Hydrophobic/hydrophilic P(VDF-TrFE)/PHEA polymer blend membranes
}

\author{
Maria N. Tamaño-Machiavello ${ }^{1}$, Benjamin Bracke ${ }^{1}$, Carlos M. Costa ${ }^{2}$, Senentxu Lanceros-Mendez ${ }^{2}$, \\ Roser Sabater i Serra ${ }^{1,3}$, José Luis Gómez Ribelles ${ }^{1,3}$ \\ ${ }^{1}$ Centre for Biomaterials and Tissue Engineering (CBIT), Universitat Politècnica de València, Camino de \\ Vera s/n, 46022 Valencia, Spain. \\ ${ }^{2}$ Centro/Departamento de Física, Universidade do Minho, 4710-057 Braga, Portugal \\ ${ }^{3}$ Biomedical Research Networking Center in Bioengineering, Biomaterials and Nanomedicine (CIBER- \\ BBN), Valencia, Spain.
}

Correspondence to: M. N. Tamaño-Machiavello (E-mail: noeltm@gmail.com)

((Additional Supporting Information may be found in the online version of this article.))

\begin{abstract}
Polymer blend membranes have been obtained consisting of a hydrophilic and a hydrophobic polymers distributed in co-continuous phases. In order to obtain stable membranes in aqueous environments, the hydrophilic phase is formed by a poly(hydrohyethyl acrylate), PHEA, network while the hydrophobic phase is formed by poly(vinylidene fluoride-co-trifluoroethylene) P(VDF-TrFE). To obtain the composites, in a first stage, P(VDF-TrFE) is blended with poly(ethylene oxyde) (PEO), the latter used as sacrificial porogen. $\mathrm{P}(\mathrm{VDF}-\mathrm{TrFE}) / \mathrm{PEO}$ blend membranes were prepared by solvent casting at $70^{\circ}$ followed by cooling to room temperature. Then PEO is removed from the membrane by immersion in water obtaining a P(VDF-TrFE) porous membrane. After removing of the PEO polymer, a P(VDF-TrFE) membrane results in which pores are collapsed. Nevertheless the pores reopen when a mixture of hydroxethyl acrylate (HEA) monomer, ethyleneglycol dimethacrylate (as crosslinker) and ethanol (as diluent) is absorbed in the membrane and subsequent polymerization yields hybrid hydrophilic/hydrophobic membranes with controlled porosity. The membranes are thus suitable for lithium-ion battery separator membranes and/or biostable supports for cell culture in biomedical applications.
\end{abstract}

KEYWORDS: Porous membrane, P(VDF-TrFE), PHEA hydrogel, hydrophilic, hydrophobic 


\section{INTRODUCTION}

Porous membranes have been used in a wide range of industrial applications including filtration, sensing, biomedical and energy applications ${ }^{1,2}$. Membranes can be made of different materials, such as, polymers ${ }^{3}$, inorganic materials ${ }^{4}$, metals ${ }^{5}$ and, composites ${ }^{6}$. Taking into account the variety and advantages of the polymers, polymer membranes are increasingly being used in a wide range of industrial applications ${ }^{7}$. In the polymer membrane technology, factors such as solvent, non-solvent, polymer type, concentration, preparation technique (template-leading, electrospinning, dip-coating, etc), and other processing parameters (temperature, humidity, pressure, etc) are very important for control of its characteristics and properties ${ }^{1,8}$. In relation to the preparation techniques, the most used polymer membrane preparation techniques are immersion, thermally induced phase separation (TIPS) and vapour induced phase separation (VIPS), etc ${ }^{9}$, 10

Poly(vinylidene fluoride), PVDF and its copolymers (poly(vinylidene fluoride-cotrifluoroethylene) $\mathrm{P}(\mathrm{VDF}-\mathrm{TrFE})$, poly(vinylidene fluoride-co-hexafluoropropene), P(VDF-HFP) included in the fluoropolymers class, are very used in the production of membranes for different areas: biomedical ${ }^{11-13}$, filtration ${ }^{14,15}$ and energy applications ${ }^{16-18}$. The copolymer P(VDF-TrFE) has been widely studied in the last few years aiming tailoring properties and microstructure for these applications ${ }^{17,19}$.

For specific molar ratios between VDF and TrFE, the semi-crystalline copolymer P(VDF-TrFE), shows a polar ferroelectric transplanar chain conformation similar to that of the $\beta$-phase of PVDF ${ }^{20,21}$. In this chain conformation the P(VDFTrFE) copolymer exhibits the ferroelectric (FE)paraelectric (PE) phase transition at a Curie temperature, Tc, below the melting temperature, $\mathrm{Tm}$. The enlargement of the crystal unit cell increases the inter-planar distance within the ferroelectric phase which consequently reduces the interaction between the units and the dipole-dipole interactions, resulting in a lower Curie temperature 22,23 .

Several methods have been used to obtain a porous membrane made of PVDF or P(VDFTrFE), including immersion precipitation, precipitation from the vapor phase, electrospinning and thermally induced phase separation (TIPS) ${ }^{24,25}$. By the TIPS method, it was reported the possibility to tailor the porous structure of the membrane through solvent evaporation at different temperatures and polymer-solvent ratios ${ }^{24}$. It was proved that the porous structure within the membranes is determined by the volume fractions of the polymer/solvent and by the temperature at which the crystallization occurs. Thus, the porosity of $\mathrm{P}(\mathrm{VDF}-\mathrm{TrFE})$ membranes is higher when crystallized at room temperature and with decreasing polymer concentration. Furthermore, interconnectivity between these pores is possible through the space between the microspheres that form the pore walls ${ }^{24,26}$.

Blends of $\mathrm{P}(\mathrm{VDF}-\mathrm{TrFE}) / \mathrm{PEO}$ were investigated for solid state batteries, where solid polymer electrolytes are used as separators and parameters such as pore size/porosity, wettability, thermal and chemical stability determine their performance 27,28. The preparation of $\mathrm{P}(\mathrm{VDF}-\mathrm{TrFE}) / \mathrm{PEO}$ blends was reported in ${ }^{28}$. Blends with varying compositions were prepared by dissolving both PEO and P(VDF-TrFE) in dimethyl formamide at $60{ }^{\circ} \mathrm{C}$ with a polymer/solvent ratio of $15 / 85$. When the film is obtained by casting from the solution at $70^{\circ} \mathrm{C}$, the $\mathrm{P}(\mathrm{VDF}-\mathrm{TrFE})$ crystallizes from the melt in the electroactive $\beta$-phase, and when cooled to room temperature, the PEO is crystallized into the confined spaces left by the P(VDF-TrFE) crystals.

Hydrogels as poly(hydroxyethyl acrylate), PHEA, have been proposed for different biomedical applications due to its excellent biocompatibility and water permeation properties ${ }^{29}$. Free radical polymerization in the presence of different solvents such as water, methanol or ethanol yields porous membranes in which the pore architecture depend on the 
amount and solubility parameters of the solvent 30,31

The objective of this work is to produce porous P(VDF-TrFE) /PHEA membranes for different applications such as cell culture supports for biomedical engineering, battery separators and filtrations membranes by a two-step method. The first step consists in the formation of a phase separated P(VDF-TrFE) / PEO blend by casting from the solution of both components in a common solvent, and then the substitution of PEO by PHEA by dissolving PEO in water and subsequently polymerize microporous or nanoporous PHEA in the space left by PEO in the $P(V D F-T r F E)$ membrane. First, the best method of polymerization was evaluated, and then the influence of increasing amounts of ethanol, added to the polymerizing mixtures, was studied.

\section{EXPERIMENTAL}

\section{Preparation of $\mathrm{P}($ VDF-TrFE)/PEO membranes}

$\mathrm{P}$ (VDF-TrFE) (Mw=350 kDa) and PEO (Mw $=100 \mathrm{kDa}$ ) were acquired from Solvay and Polysciences, respectively. P(VDF-TrFE) and PEO with 50/50 weight ratio were diluted in N,Ndimethylformamide to obtain a $15 / 85$ ratio of solute-solvent. The choice of this composition is due to the fact that larger PEO contents in the polymer blend lead to fragile membranes and removing PEO yields a discontinuous material. This mixture was magnetically stirred at $60{ }^{\circ} \mathrm{C}$ during 24 hours until a homogeneous and transparent sample was obtained. Then the mixture was deposited on clean glass substrates at $70{ }^{\circ} \mathrm{C}$ in order to let solvent evaporation occur. The evaporation continued for 2 hours at atmospheric pressure and subsequently 3 hours at $10-2 \mathrm{~mm} \mathrm{Hg}$ at $70{ }^{\circ} \mathrm{C}$. Finally, the membrane was cooled to room temperature.

\section{Removing PEO}

As PEO is a hydrophilic component, it can be removed by immersing in water. The membranes were immersed and stirred during
7 days, while the water was changed every day. After 7 days, the samples were dried in open air during one day and subsequently dried in vacuum at $40{ }^{\circ} \mathrm{C}$ during one more day.

The amount of PEO removed from the PVDFTrFE/PEO membranes, was determined by weighing before and after washing the sample in water. Volume fraction of PEO in the membrane was calculated from the mass ratio and the density data of both components (data by manufacturer: density of PVDF-TrFE: 1,917 $\mathrm{g} / \mathrm{ml}$; density of PEO: 1,130 g/ml)

\section{PHEA Polymerization}

Monomer mixtures of hydroxyethyl acrylate HEA were prepared under stirring at room temperature for one hour. Reactant mixtures contained $1 \%$ by weight ethylene glycol dimethacrylate (EGDMA) (cross-linking agent) and $0.5 \%$ by weight of benzoyl peroxide (BPO) (initiator), were performed with a varying amount of ethanol ranging between 0 and $60 \%$ by weight. All percentages refer to the monomers weight. P(VDF-TrFE) membrane was immersed in the monomer mixtures during 6 hours to allow monomer mixture absorption. Then, the samples were isolated in a Teflon mold and polymerization took place at $60^{\circ} \mathrm{C}$ for 24 hours and after that, at $90{ }^{\circ} \mathrm{C}$ for another 24 hours. The samples were dried for 24 hours at room temperature and then placed in ethanol at $80^{\circ} \mathrm{C}$ to wash for 5 days. Finally, the samples were dried in vacuum for 3 days at $60^{\circ} \mathrm{C}$.

\section{Characterization techniques}

A JSM6300 from JEOL was used for CryoScanning electron microscopy (Cryo-SEM). Samples were immersed for 24 hours in water and then inside the instrument they were cooled to $-90{ }^{\circ} \mathrm{C}$ and cryo fractured. Water sublimation in vacuum took place for 30 minutes, and then the surface was coated with a layer of gold before the images were obtained. Images were taken at acceleration voltage $15 \mathrm{kV}$.

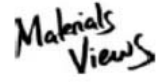


Differential Scanning calorimetry (DSC) was carried out with a DSC 8000 of Perkin-Elmer under a flowing nitrogen atmosphere between $40{ }^{\circ} \mathrm{C}$ and $200{ }^{\circ} \mathrm{C}$ at a heating rate of $20^{\circ} \mathrm{C}$ min- 1 for both cooling and heating. Samples weight was between 5 to $10 \mathrm{mg}$.

Fourier transform infrared spectroscopy analysis (FTIR) was performed by a Thermo Nicolet Nexus in Attenuated Total Reflectance (ATR) mode. Infrared spectra were obtained of the membranes for wavelengths from $600 \mathrm{~cm}-1$ to $4000 \mathrm{~cm}-1$ with a resolution of $4 \mathrm{~cm}-1$.

The contact angle of deionized water on the different samples was measured with the equipment "Dataphysics OCA 20" delivered by Dataphysics $\mathrm{GmBH}-$ Neurtek S.A. Images were taken always $30 \mathrm{~s}$ after the placement of the droplet on the sample.

\section{RESULTS}

\section{Removal of PEO}

As it has been shown in ${ }^{27,28}$, casting at $70{ }^{\circ} \mathrm{C}$ from the homogeneous solution of PEO and $\mathrm{P}$ (VDF-TrFE) in DMF yields a homogeneous polymer blend in which $\mathrm{P}$ (VDF-TrFE) is semicrystalline and molten PEO occupies the spaces among P(VDF-TrFE) domains. On further cooling to room temperature PEO crystallizes as well. The volume fraction of PEO in the P(VDFTrFE)/PEO membrane was found to be $63 \pm 1 \%$ for a 50/50 wt\% composition. When the membrane was subsequently immersed in water during 7 days, it was established by weighing that the weight loss was $52 \pm 1$ wt \%. These results show that some P(VDF-TrFE) is lost as well, even if this component is not soluble in water and must be ascribed to the delivery of small P(VDF-TrFE) domains dispersed in the PEO phase, that leave the sample when the PEO that surrounds them are dissolved. To check if PEO is completely removed from the membrane, DSC and FTIR scans were performed (Figure 1).
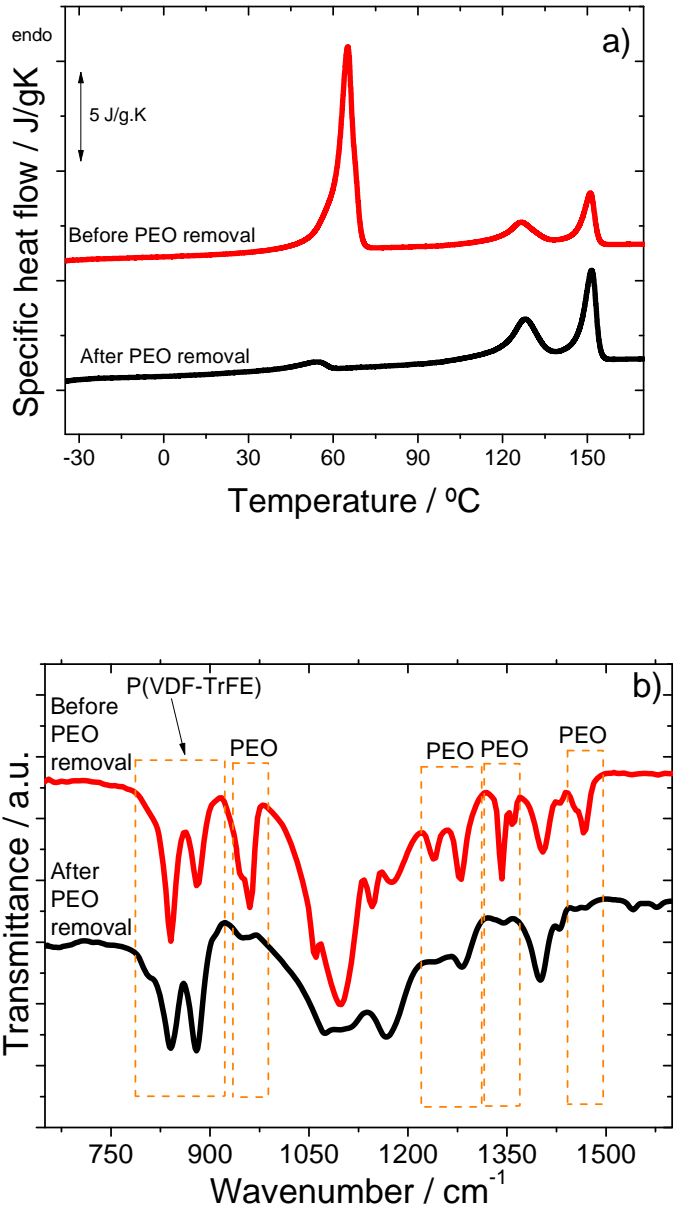

FIGURE 1 a) DSC thermogram and b) Infrared spectra of PVDF-TrFE/PEO samples before and after $P E O$ removal.

Independently of PEO removal, the vibrational modes of $\mathrm{P}(\mathrm{VDF}-\mathrm{TrFE})$ polymer were observed at the main absorption bands at 851,886 , and $1402 \mathrm{~cm}^{-1}$, corresponding to the all-trans conformation of the polymer ${ }^{21,32}$. The characteristic bands of PEO are clearly observed in the infrared spectrum of the original P(VDFTrFE)/PEO blend in (figure $1 \mathrm{~b}$ ): the vibrations bands at wavelengths of $964 \mathrm{~cm}^{-1}, 1343 \mathrm{~cm}^{-1}$ and $1468 \mathrm{~cm}^{-1}$ represent vibrational modes of $\mathrm{CH}_{2}$ rocking, trans-zigzag conformation, $\mathrm{CH}_{2}$ 
wagging, trans-zigzag conformation and $\mathrm{CH}_{2}$ scissoring ${ }^{33}$. Nevertheless these bands are not detected after washing, supporting the removal of PEO.

The DSC thermograms of the P(VDF-TrFE)/PEO blend show three endothermic peaks (Figure 1a). The lowest temperature peak corresponds to the melting of PEO around $55-68{ }^{\circ} \mathrm{C}^{34}$, the second peak at $\sim 11{ }^{\circ} \mathrm{C}$ corresponds to the FE$\mathrm{PE}$ transition (Curie transition) of P(VDF-TrFE) and the peak around $145^{\circ} \mathrm{C}$ corresponds to the melting of the PE phase of P(VDF-TrFE) ${ }^{28}$ (Figure 1a). Interestingly, while in the first heating scan recorded after washing, the melting peak of PEO is completely absent as expected (figure 1a), the second heating scan performed after cooling at $20{ }^{\circ} \mathrm{C} / \mathrm{min}$ after the first one, shows a small endotherm peak at around $55{ }^{\circ} \mathrm{C}$ (Figure 1a). This peak must be ascribed to small traces of disperse domains of PEO remaining in the membrane. This would mean that a very small (but not quantifiable with enough accuracy with our techniques) amount of PEO is isolated in the P(VDFTrFE)/PEO blend in regions that are not accessible to water.

Extraction of PEO yields also a reduction in the volume of the sample of $23 \pm 1 \%$ and the sample turned white after PEO removal. Comparing this volume reduction with the initial volume fraction of PEO in the blend, it can be deduced that although some porosity remains in the $P$ (VDF-TrFE) membrane, it is not as large as expected. In fact, a SEM picture of the cross-section of the cryo-fractured sample after PEO extraction shows a smooth surface in which porosity cannot be appreciated (Figure 2).

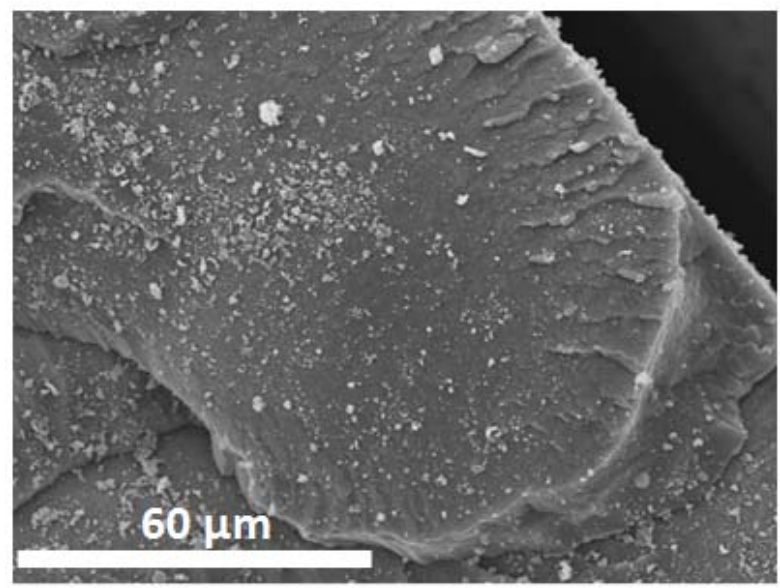

FIGURE 2 Cryo-SEM pictures of a cross section of cryo-fractured P(VDF-TrFE) membrane after PEO extraction

\section{P(VDF-TrFE)/PHEA hybrid membranes}

Although the membrane did not absorb water or ethanol, it was able to swell when immersed in the liquid monomer mixture (HEA, EGDMA, PBO). After swelling to equilibrium, the weight increase of the sample was $52 \pm 1 \%$. It is worth noting that $\mathrm{P}$ (VDF-TrFE) is not able to absorb HEA. A P(VDF-TrFE) film prepared without PEO was immersed in liquid HEA for 6 hours at room temperature and absorbed less than $0.1 \%$ by weight of monomer. Thus, the weight increment of the membrane has to be ascribed to HEA monomer filling the pores, indicating that the pores that collapsed after PEO extraction reopen during monomer absorption. Other methods to introduce the monomer into the membrane pores, such as injection of HEA monomer on the surface and performing vacuum cycles, were less effective than simple immersion in the monomer mixture.

Monomeric mixtures containing varying amounts of ethanol as diluent were prepared in order to modulate water sorption capacity of the membranes. As the ethanol content increases, the amount of PHEA into the resulting $\mathrm{P}(\mathrm{VDF}-\mathrm{TrFE}) / \mathrm{PHEA}$ membrane decreases, but in addition, the PHEA phase becomes microporous due to the phase 
separation between PHEA and ethanol during PHEA polymerization. Polymerization took place at $60{ }^{\circ} \mathrm{C}$ for 24 hours and then the temperature was increased to $90{ }^{\circ} \mathrm{C}$ to end monomer conversion and evaporate volatiles. Table 1 shows the percentage of PHEA in the P(VDFTrFE)/PHEA membranes obtained by weighing after polymerization and drying of the sample. When the ratio HEA/ethanol increases, the total amount of PHEA in the membrane decreases and when this ratio reach $40 / 60$ by weight, polymerization of HEA does not occur and all the monomers and solvent evaporate.

TABLE 1 Influence of ethanol on weight increase of PHEA inside the membrane

$\begin{array}{cc}\text { amount of ethanol (\%) } & \text { weight increase (wt\%) } \\ 0 & 62 \\ 20 & 23 \\ 40 & 16 \\ 60 & 0\end{array}$

Figure 3 shows the SEM images of cryofractured samples obtained with different ethanol contents. Polymerization of PHEA took place in sealed Petry dishes. The top side of the membrane appears in the left bottom corner of the pictures of Figure 3 . Interestingly enough, when the PHEA phase was obtained introducing only HEA and EGDMA, the resulting membrane does not show porosity; it appears as a continuous phase separated polymer blend in which P(VDF-TrFE) and PHEA domains coexist (Figures $3 a, 3 b$ ). The appearance of the sample obtained with $20 \%$ ethanol shows the same structure in most of the cross section but some elongated large pores appear close to the surface (Figures 3c, $3 d$ ). These large pores (figure $3 c$ ) grow in number with increasing amount of ethanol, in fact when ethanol content is $40 \%$, these large pores fill the whole membrane (Figures 3e, 3f). As a consequence of the porosity, the thickness of the sample increases with ethanol content.
Figures $3 a, 3 c$ and $3 e$ were taken with the same magnification and while the full thickness of the membrane can b observed in Figures $3 a$ and 3c, in the case of the membrane obtained with $40 \%$ ethanol, only a portion of the cross-section is shown.

In the sample obtained with $60 \%$ ethanol the porous structure is also formed although with a different morphology with smaller and uniform pores (Figures 3g, 3h).
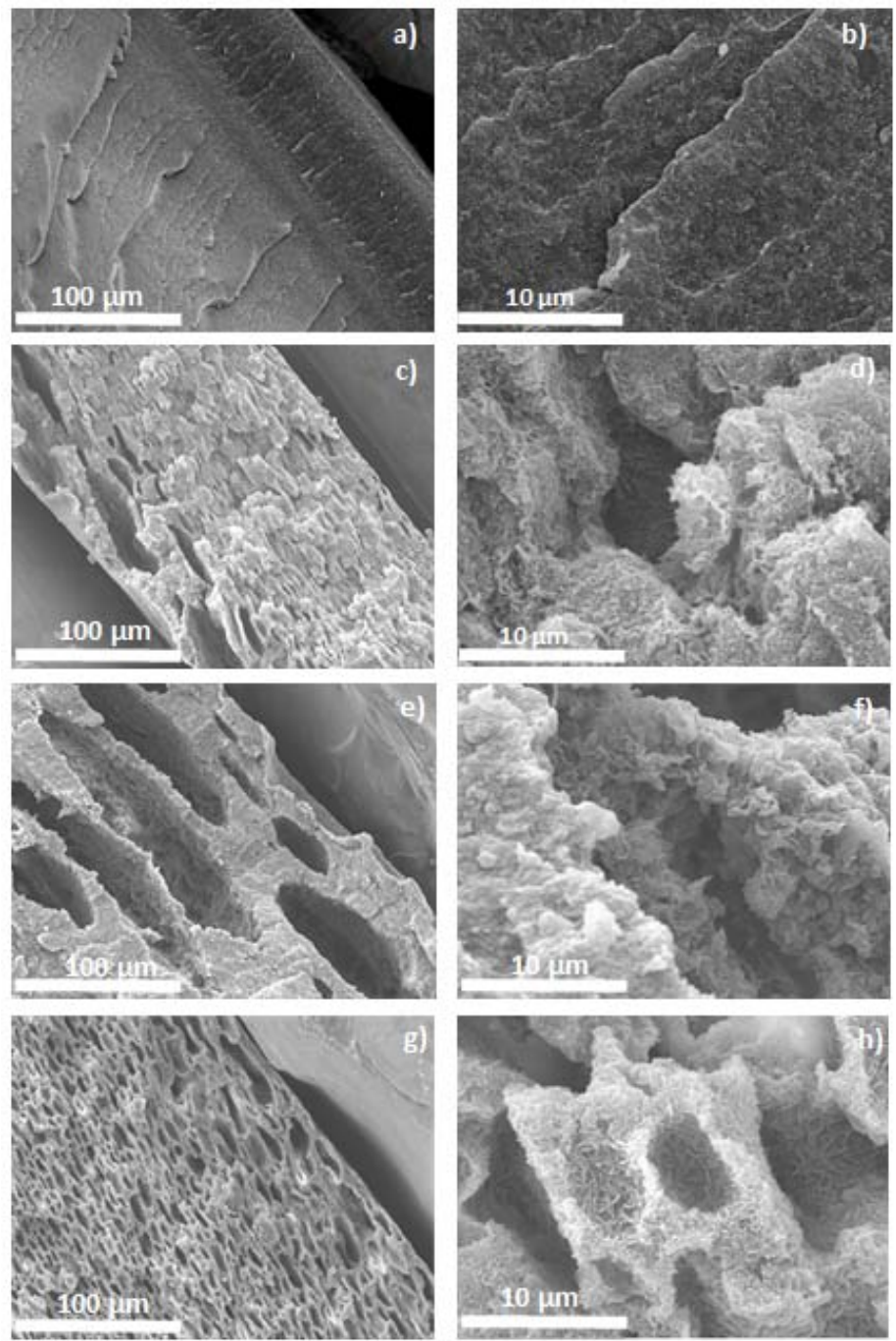

FIGURE 3 SEM images of a polymerized P(VDFTrFE) sample a different magnification of $500 x$ and $3500 x$, respectively for: (a-b) $0 \%$ ethanol (c-d) $20 \%$ ethanol, (e-f) $40 \%$ ethanol and (g-h) $60 \%$ ethanol. 
The formation of this porous structure can be ascribed to the foaming effect of the vapour pressure of ethanol when temperature increases during PHEA polymerization, due to the auto acceleration of the exothermal free radical polymerization that deliver an important amount of heat locally, or even when temperature was increased to dry the membrane after polymerization. At the surface of the sample only a thin layer of $\mathrm{P}$ (VDF-TrFE) is available to sustain the internal pressure exerted by the vapor of ethanol and thus, pores appear first in this zone of the sample, as shown in Figure 3c. When the ethanol content grows, pores in the whole membrane appear as shown in Figure 3e. In the case of the membrane prepare with HEA monomer diluted with $60 \%$ ethanol, foaming is produced only because the increase of temperature since no polymerization of PHEA takes place as demonstrated by the weight increase of the membrane. Then, still porosity appears, but now the pores are smaller and more homogeneously distributed (Figure 3g).

Figures $3 b, 3 d, 3 f$ and $3 \mathrm{~h}$ show details at larger magnifications of the $m$ ost compacted zones of the cross section, i.e., the walls of the large pores described above. These pictures show that these compacted zones are also microporous when HEA monomer is diluted with ethanol.

In this way, the resulting membrane is macroporous and contains PHEA filling a part of the volume of these pores that results in an important influence on water sorption and surface tension properties.

The presence of PHEA polymer inside the $\mathrm{P}$ (VDF-TrFE) membrane was confirmed through the FTIR spectrum shown in figure 4. The characteristic vibration bands for PHEA are detected in figure 4 at $1719 \mathrm{~cm}^{-1}, 1394 \mathrm{~cm}^{-1}$ and at $1162 \mathrm{~cm}^{-1}$, which represent respectively the carbonyl band, $\mathrm{OH}-$ bending and the ester group of the polymer ${ }^{35-37}$.

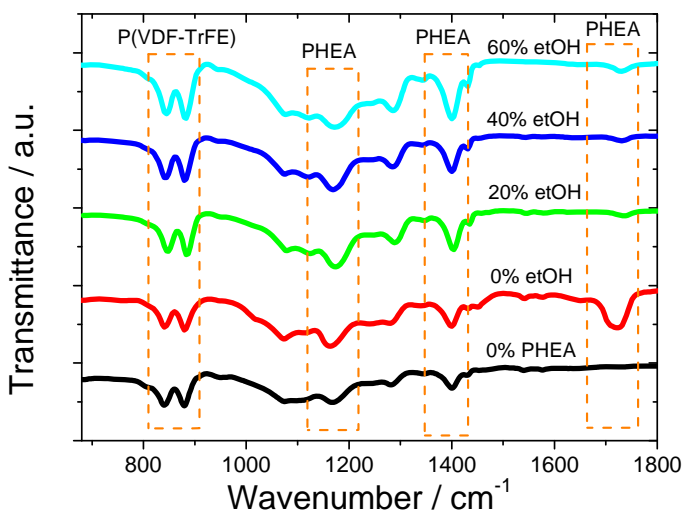

FIGURE 4 FTIR spectra of P(VDF-TrFE) membranes after polymerization with PHEA and different amounts of ethanol as solvent

Independently of the ethanol content, it is observed at $851 \mathrm{~cm}^{-1}$ and $886 \mathrm{~cm}^{-1}$ the characteristic vibration bands of the polar phase of $P(V D F-T r F E){ }^{21}$. At $1291 \mathrm{~cm}^{-1}$, another vibration band of $\mathrm{P}$ (VDF-TrFE) is observed, which correspond to CC symmetric stretching ${ }^{21}$. This fact suggests that the polymerization of HEA inside the pores of the membrane has no influence on the crystal structure of P(VDF-TrFE) polymer.

For all polymerized samples shown in figure 4, the bands at $1162 \mathrm{~cm}^{-1}$ and $1719 \mathrm{~cm}^{-1}$ are observed. They represent the carbonyl band and ester group, respectively, vibration bands characteristics of PHEA polymer that indicate the presence of PHEA within the pores.

The presence of the PHEA bands in the sample polymerized with $60 \%$ ethanol, in which no significant amount of PHEA was detected by weighing, can be due to the use of ATR in the FTIR experiments. The spectra recorded are representative of the chemical properties of the material close to the surface. Perhaps a small amount of PHEA polymerizes in this region due to the surface evaporation of ethanol, which 
increases the concentration of monomer in this region, allowing polymerization.

PHEA is an amorphous polymer and the glass transition of the PHEA phase within the membrane can be observed in the heating DSC thermograms (Figure 5). The glass transition temperature, between 9 and $13{ }^{\circ} \mathrm{C}$, is that expected for PHEA ${ }^{29-31,38}$. It is worth noting the absence of glass transition in the sample polymerized with $60 \%$ ethanol.

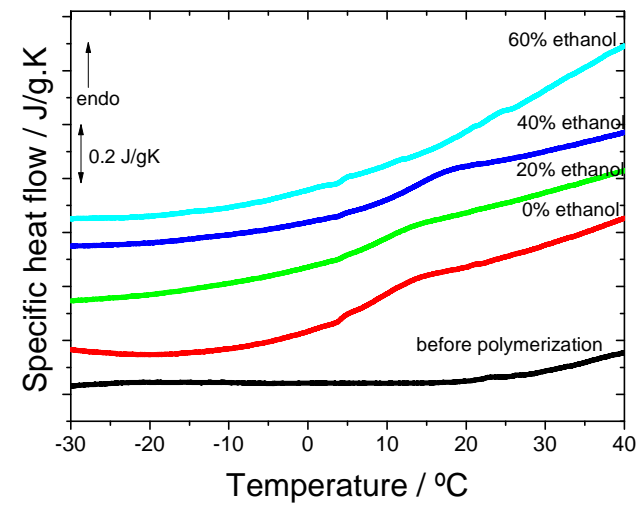

FIGURE 5 DSC of the hybrid membranes in which HEA was polymerized with different amounts of ethanol in the monomer mixture

Water absorption depends on the amount of the hydrophilic PHEA component contained in the membrane, but also on the presence of pores in the membrane (Figure 6). This explains why the membrane in which HEA was polymerized with $20 \%$ ethanol absorbs more water than the one in which HEA was polymerized without diluents, in spite that the PHEA content of the membrane is smaller. On the other hand, larger diluent contents yields less water sorption because the PHEA content is smaller, in spite of the porosity. The case of the sample polymerized with $60 \%$ ethanol shows that although the porosity is high, water is not able to invade the pore structure because of its hydrophobicity.

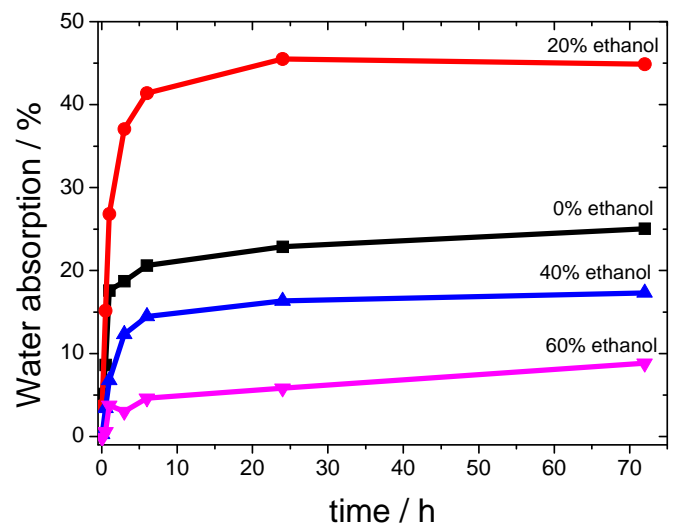

FIGURE 6 Water absorption for samples in which PHEA was polymerized with different amounts of ethanol in the monomer mixture

Table 2 shows the contact angle of samples before and after polymerization with different amounts of ethanol in the monomer mixture. The contact angle values shown indicate that the surface of the samples after polymerization is more hydrophobic than the sample before polymerization, but the difference is less to $20^{\circ}$. The amount of ethanol does not seem to have a significant influence regarding the contact angle value for the membranes polymerized with $0 \%$, $20 \%$ and $40 \%$ ethanol. However, the result of the one polymerized with $60 \%$ ethanol seems slightly more hydrophobic than the other membranes, suggesting that less PHEA is present within that membrane than in the others.

Thus, porous membranes with high water sorption capacity, but whose surface is hydrophobic, can be produced with the procedure proposed in this work. In addition the hydrophilic phase is formed by a crosslinked hydrogel, and hence it is stable inside the membrane when immersed in aqueous media. 
TABLE 2 - Contact angle of samples before and after polymerization

$\begin{array}{cc}\text { Sample } & \text { Contact angle }\left(^{\circ}\right) \\ \text { Before polymerization } & 90 \pm 3 \\ 0 \% \text { ethanol } & 99 \pm 3 \\ 20 \% \text { ethanol } & 103 \pm 3 \\ 40 \% \text { ethanol } & 100 \pm 3 \\ 60 \% \text { ethanol } & 107 \pm 3\end{array}$

\section{CONCLUSIONS}

P(VDF-TrFE) /PEO 50/50 blends are cocontinuous, thus PEO can be extracted by immersion in water, and therefore PEO can be used as a sacrificial porogen material. Nevertheless, the volume occupied by PEO does not produce the same volume of pores in the resulting $P(V D F-T r F E)$ membrane, that is not able to absorb characteristic non solvents of P(VDF-TrFE), such as water or ethanol. Furthermore, after observing cryo-SEM images, the resulting material does not seem porous. However, the membrane is capable of absorbing the monomer HEA after immersion in the monomer for long periods and even mixtures of HEA with ethanol. The ethanol evaporation during polymerization of HEA produces a macroporous structure due to the foaming effect of the vapour pressure of ethanol. The result is a porous membrane with a cross-linked PHEA hydrophilic phase and a hydrophobic and electroactive P(VDF-TrFE) phase, in which the surface has hydrophobic properties in spite of the high water sorption capacity.

\section{ACKNOWLEDGEMENTS}

This work was funded by the Spanish Ministry of Economy and Competitiveness (MINECO) through the project MAT2013-46467-C4-1-R (including the FEDER financial support). The work of the Portuguese group was funded by FEDER funds through the "Programa Operacional Factores de Competitividade e COMPETE" and by national funds from
Fundação para a Ciência e a Tecnologia, in the framework of the strategic project Strategic Project PEST-C/FIS/UI607/2014. The authors also thank funding from Matepro - Optimizing Materials and Processes", ref.NORTE-07-0124FEDER-000037", co-funded by the "Programa Operacional Regional do Norte" (ON.2 e O Novo Norte), under the "Quadro de Referência Estratégico Nacional" (QREN), through the "Fundo Europeu de Desenvolvimento Regional" (FEDER), and grant SFRH/BD/68499/2010 (C.M.C.).

CIBER-BBN is an initiative funded by the VI National R\&D\&I Plan 2008-2011, Iniciativa Ingenio 2010, Consolider Program, CIBER Actions and financed by the Instituto de Salud Carlos III with assistance from the European Regional Development Fund. MNTM acknowledges the Programa de Ayuda de Investigación y Desarrollo (PAID) of the Universitat Politècnica de València for her doctoral grant. The authors acknowledge the assistance and advice of Electron Microscopy Service of the UPV

\section{REFERENCES AND NOTES}

1. Mulder, M. Basic Principles of Membrane Technology; Springer, 1996.

2. Sirkar, K. K. Industrial \& Engineering Chemistry Research 2008, 47, 52505266.

3. Ulbricht, M. Polymer 2006, 47, 22172262.

4. Moore, T. T.; Koros, W. J. Journal of Molecular Structure 2005, 739, 87-98.

5. Zhao, J.; Deng, Q.; Bachmatiuk, A.; Sandeep, G.; Popov, A.; Eckert, J.; Rümmeli, M. H. Science 2014, 343, 1228-1232.

6. Xu, Z.-I.; Yu, L.-y.; Han, L.-f. Frontiers of Chemical Engineering in China 2009, 3, 318-329.

7. Yang, Q.; Adrus, N.; Tomicki, F.; Ulbricht, M. Journal of Materials Chemistry 2011, 21, 2783-2811.

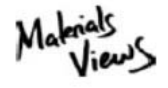

WWW.MATERIALSVIEWS.COM 
8. Guillen, G. R.; Pan, Y.; Li, M.; Hoek, E. M. V. Industrial \& Engineering Chemistry Research 2011, 50, 3798-3817.

9. Matsuura, T. Synthetic Membranes and Membrane Separation Processes; Taylor \& Francis, 1993.

10. Li, N. N.; Fane, A. G.; Ho, W. S. W.; Matsuura, T. Advanced Membrane Technology and Applications; Wiley, 2011.

11. Zhu, L.-P.; Yu, J.-Z.; Xu, Y.-Y.; Xi, Z.-Y.; Zhu, B.-K. Colloids and Surfaces B: Biointerfaces 2009, 69, 152-155.

12. Boschin, F.; Blanchemain, N.; Bria, M.; Delcourt-Debruyne, E.; Morcellet, M.; Hildebrand, H. F.; Martel, B. Journal of Biomedical Materials Research Part A 2006, 79A, 78-85.

13. Ribeiro, C.; Moreira, S.; Correia, V.; Sencadas, V.; Rocha, J. G.; Gama, F. M.; Gomez Ribelles, J. L.; Lanceros-Mendez, S. RSC Advances 2012, 2, 11504-11509.

14. Park, S.-J.; Cheedrala, R.; Diallo, M.; Kim, C.; Kim, I.; Goddard, W., III. Journal of Nanoparticle Research 2012, 14, 114.

15. Buonomenna, M. G.; Lopez, L. C.; Favia, P.; d'Agostino, R.; Gordano, A.; Drioli, E. Water Research 2007, 41, 4309-4316.

16. Alwin, S.; Bhat, S. D.; Sahu, A. K.; Jalajakshi, A.; Sridhar, P.; Pitchumani, S.; Shukla, A. K. Journal of The Electrochemical Society 2011, 158, B91B98.

17. Costa, C. M.; Rodrigues, L. C.; Sencadas, V.; Silva, M. M.; Rocha, J. G.; LancerosMéndez, S. Journal of Membrane Science 2012, 407-408, 193-201.

18. Costa, C. M.; Silva, M. M.; LancerosMendez, S. RSC Advances 2013, 3, 11404-11417.

19. Navid, A.; Lynch, C. S.; Pilon, L. Smart Materials and Structures 2010, 19, 055006 .

20. Nalwa, H. S. Ferroelectric Polymers: Chemistry: Physics, and Applications; Taylor \& Francis, 1995.
21. Martins, P.; Lopes, A. C.; LancerosMendez, S. Progress in Polymer Science 2014, 39, 683-706.

22. Mao, D.; Gnade, B. E.; Quevedo-Lopez, M. A. Ferroelectric Properties and Polarization Switching Kinetic of Poly (vinylidene fluoride-trifluoroethylene) Copolymer, 2011.

23. Lutkenhaus, J. L.; McEnnis, K.; Serghei, A.; Russell, T. P. Macromolecules 2010, 43, 3844-3850.

24. California, A.; Cardoso, V. F.; Costa, C. M.; Sencadas, V.; Botelho, G.; GómezRibelles, J. L.; Lanceros-Mendez, S. European Polymer Journal 2011, 47, 2442-2450.

25. Gu, M.; Zhang, J.; Wang, X.; Tao, H.; Ge, L. Desalination 2006, 192, 160-167.

26. Ferreira, A.; Silva, J.; Sencadas, V.; Ribelles, J. L. G.; Lanceros-Méndez, S. Macromolecular Materials and Engineering 2010, 295, 523-528.

27. Costa, C. M.; Nunes-Pereira, J.; Rodrigues, L. C.; Silva, M. M.; Ribelles, J. L. G.; Lanceros-Méndez, S. Electrochimica Acta 2013, 88, 473-476.

28. Costa, C. M.; Machiavello, M. N. T.; Ribelles, J. L. G.; Lanceros-Méndez, S. Journal of Materials Science 2013, 48, 3494-3504.

29. Kyritsis, A.; Pissis, P.; Ribelles, J. L. G.; Pradas, M. M. Polymer Gels and Networks 1995, 3, 445-469.

30. Monleón Pradas, M.; Gómez Ribelles, J. L.; Serrano Aroca, A.; Gallego Ferrer, G.; Suay Antón, J.; Pissis, P. Polymer 2001, 42, 4667-4674.

31. Serrano Aroca, A.; Campillo Fernández, A. J.; Gómez Ribelles, J. L.; Monleón Pradas, M.; Gallego Ferrer, G.; Pissis, P. Polymer 2004, 45, 8949-8955.

32. Faria, L. O.; Moreira, R. L. Journal of Polymer Science Part B: Polymer Physics 2000, 38, 34-40.

33. Shieh, Y.-T.; Liu, K.-H. Journal of Polymer Science Part B: Polymer Physics 2004, 42, 2479-2489. 
34. Afifi-Effat, A. M.; Hay, J. N. Journal of the Chemical Society, Faraday Transactions 2: Molecular and Chemical Physics 1972, 68, 656-661.

35. Liu, Z.; Zhu, S.; Li, Y.; Li, Y.; Shi, P.; Huang, Z.; Huang, X. Polymer Chemistry 2015.

36. Jiang, X.; Jiang, X.; Lu, G.; Feng, C.; Huang, X. Polymer Chemistry 2014, 5, 4915-4925.
37. Liu, Y.-Y.; Lü, J.; Shao, Y.-H. Macromolecular Bioscience 2006, 6, 452-458.

38. Castilla Cortázar, I.; Vidaurre, A.; Gallego Ferrer, G.; Monleón Pradas, M.; Gómez Ribelles, J. L.; Meseguer Dueñas, J. M. Journal of Non-Crystalline Solids 2001,

287, 130-134. 


\section{GRAPHICAL ABSTRACT}

Maria N. Tamaño-Machiavello, Benjamin Bracke, Carlos M. Costa, Senenxtu Lanceros-Méndez, Roser Sabater i Serra, José Luis Gómez Ribelles

\section{HYDROPHOBIC/HYDROPHILIC P(VDF-TrFE)/PHEA POLYMER BLEND MEMBRANES}

Poly(vinylidene fluoride-co-trifluoroethylene)/poly(hydroxyethyl acrylate), P(VDF-TrFE)/PHEA membranes, that are suitable for applications such as battery separators, filtration membranes and cell culture supports have been investigated as a function of amounts of ethanol, added to the polymerizing mixtures. The membrane is capable of absorbing the monomer HEA after immersion in the monomer for long periods and even mixtures of HEA with ethanol. FTIR, DSC, cryo-SEM, equilibrium water content and contact angel measurement were performed for membrane characterization in function of amount of ethanol.

\section{GRAPHICAL ABSTRACT FIGURE}

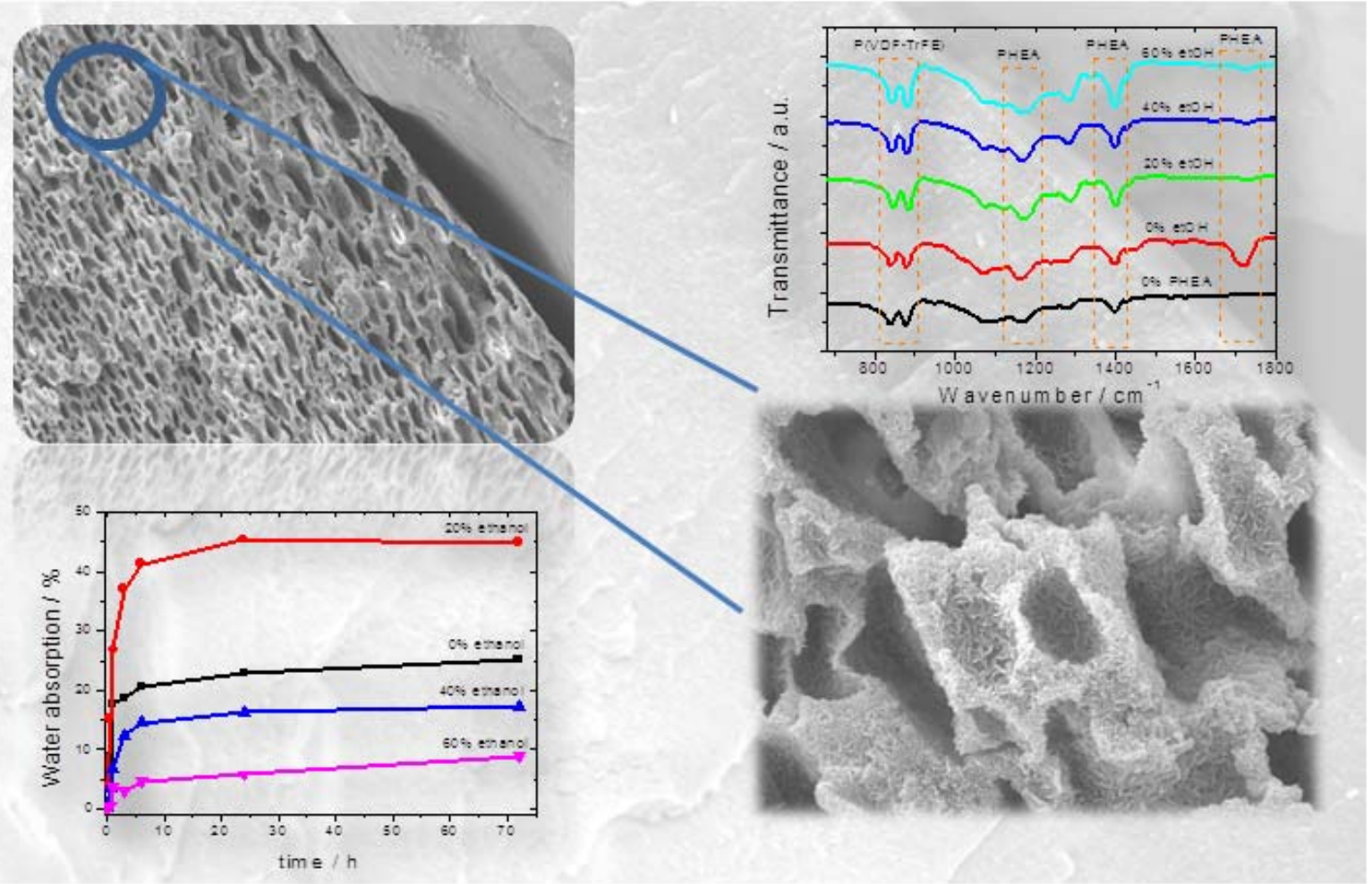

